\title{
Oversized W-band 2D Periodic Lattice Oscillator
}

\author{
A. W. Cross, A. J. MacLachlan, C. W. Robertson, L. Zhang, C. R. Donaldson, H. Yin, A. D. R. Phelps, \\ and K. Ronald \\ Department of Physics, SUPA, University of Strathclyde, Glasgow, G4 ONG, UK \\ a.w.cross@strath.ac.uk
}

\begin{abstract}
To mitigate the conventional scaling of slow-wave vacuum electronic oscillators, in which the maximum output power reduces as the frequency increases, an oversized cylindrical structure is driven by an annular electron beam. To enhance mode selectivity a two dimensional (2D) periodic lattice structure (PSL) is used. The 2D PSL consists of shallow periodic cosinusoidal perturbations in both the azimuthal and axial directions on the inner wall of a cylindrical waveguide. Analytical theory and numerical PIC simulations have been used to design the $\mathbf{W}$-band oscillator that has been constructed. The ratio of the diameter of the cylindrical cross-section of the structure to the operating wavelength is $\sim 5$. The performance of this oscillator is being measured and compared with the predictions of the numerical simulations.
\end{abstract}

Keywords-2D periodic lattice, $W$-band, oscillator, mm-waves

\section{INTRODUCTION}

The advantages provided by the application of periodic lattice structures in vacuum electronic microwave sources has been of growing interest [1-11]. Two dimensional (2D) periodic surface lattices PSLs have been used successfully in both fast-wave sources [2] and in slow-wave sources [5-14], although the details of the physical interactions that are involved differ in the two cases. In the present work the focus is on a slow wave interaction in the W-band (75-110 GHz).

\section{THEORY}

A hollow conducting cylindrical waveguide with shallow periodic cosinusoidal perturbations in both the azimuthal $\varphi$ and axial $z$ directions on the inner wall is described by the equation,

$$
r=r_{0}+\Delta r\left[\cos (m \varphi) \cos \left(k_{z} z\right)\right]
$$

where $m$ is the number of azimuthal variations, $r_{0}$ is the mean radius of the waveguide, $\Delta r$ is the amplitude of the perturbation, and $k_{z}=2 \pi / d z$, where $d z$ is the period of the perturbation along the axial z-coordinate.

The diameter of the waveguide $\left(D=2 r_{0}\right)$ is able to be made much larger than in traditional slow wave devices. In the present work the cylindrical cavity is oversized to the extent that $D / \lambda \sim 5$, where $\lambda$ is the wavelength of the $\mathrm{W}$-band radiation. A near cut-off $T M_{0, n}$ volume mode facilitates wave synchronization.

A series of cases with a range of initial parameters have been numerically modelled using the CST Studio software suite. The parameters and dimensions of the 2D PSL configuration, the radial position of the annular electron beam, its accelerating potential in steps from $60 \mathrm{kV}$ to $90 \mathrm{kV}$ and current values ranging between $100 \mathrm{~A}$ and $200 \mathrm{~A}$ have been used as inputs to the numerical model. The results of the modelling show that, as expected, such a highly overmoded

Support was received from the Leverhulme Trust Network "Advanced Research on Generation of THz and X-ray Radiation" (IN-2015-012) and from AFOSR under awards FA8555-13-1-2132 and FA9550-17-1-0095. interaction structure is sensitive to relatively small changes in the input parameters.

Outputs at $\sim 95 \mathrm{GHz}$ of MW level with over $10 \%$ electronic efficiency are predicted by the modelling with appropriately chosen values of the length of the 2D PSL interaction region and with optimum selected 2D PSL parameters $r_{0}, \Delta r, d z$, and $m$.

\section{EXPERIMENT}

W-band 2D PSL interaction structures have been constructed using two manufacturing methods (1) electrochemical deposition of copper on a cylindrical aluminum former with the aluminum subsequently removed by dissolving in strong alkali solution and (2) a 3D additive manufacturing (3D printing) technique resulting in a silver/chromium cylindrical 2D PSL. The structure used in the experimental program that is being reported here has used method (1). An earlier experiment [12] used the 3D additive manufacturing method. The computer numerically controlled (CNC) machined aluminum former is shown in Fig. 1 and the outside of the electrochemically deposited copper structure is shown in Fig. 2.

A sharpened annular graphite cathode is used to provide the annular electron beam source. The electron beam of $\sim 100 \mathrm{~ns}$ pulse duration that can be varied over the accelerating voltage range from $60 \mathrm{kV}$ to $90 \mathrm{kV}$, is guided by a strong axial magnetic field provided by a superconducting magnet and is passed through the cylindrical 2D PSL copper cavity. The superconducting magnet is one that has been used in other applications and is capable of supplying a magnetic field that can be systematically varied up to 11 tesla, although the magnetic field needed to provide electron beam transport through the cylindrical 2D PSL structure in the present experimental program is only a few tesla. The diagnostics available to characterize the electron beam include Faraday cup, witness plate and macroscopic current/voltage measurements. The mm-wave diagnostics include cut-off waveguide filters, W-band heterodyne mixer and intermediate frequency capture and analysis, as well as output mode pattern measurements.

The assembled W-band oscillator is shown in Fig. 3. More than one 2D PSL interaction structure has been constructed, so as to be able to change the structure and compare the experimental results with numerical simulations carried out for a range of structure parameters. In order to be able to easily change the 2D PSL structure the vacuum envelope of the oscillator, including the electron gun, the output waveguide, electron beam collector and the output window have been constructed so as to be demountable. The vacuum system is continually pumped and the measured vacuum pressure is $\sim 3 \times 10^{-6}$ mbar. This pressure is sufficiently low to 
allow reliable production from the sharpened graphite cold cathode of $\sim 100$ ns electron beam pulses.

\section{CONCLUSIONS}

Theory and simulations have enabled the design of an oversized $(D / \lambda \sim 5) \mathrm{W}$-band 2D PSL oscillator that uses a $2 \mathrm{D}$ PSL to provide mode rarefaction in the overmoded interaction structure. In the present research program electrodeposition of copper on an aluminum former has been used to construct the 2D PSL interaction structure. The experiment with its vacuum system, magnet and power supplies has been assembled. The necessary vacuum pressure has been achieved and the experimental program of measurements is underway.

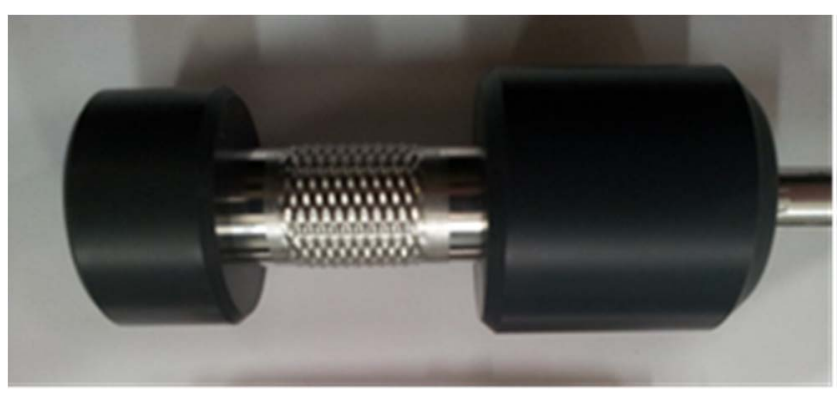

Fig. 1. CNC machined aluminum former.

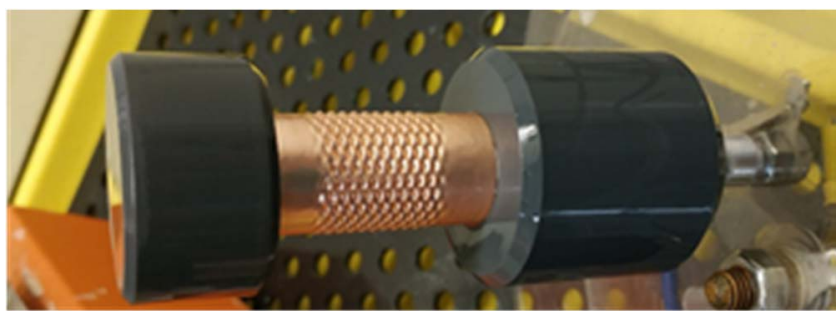

Fig. 2. Electrodeposited copper 2D cylindrical periodic structure.

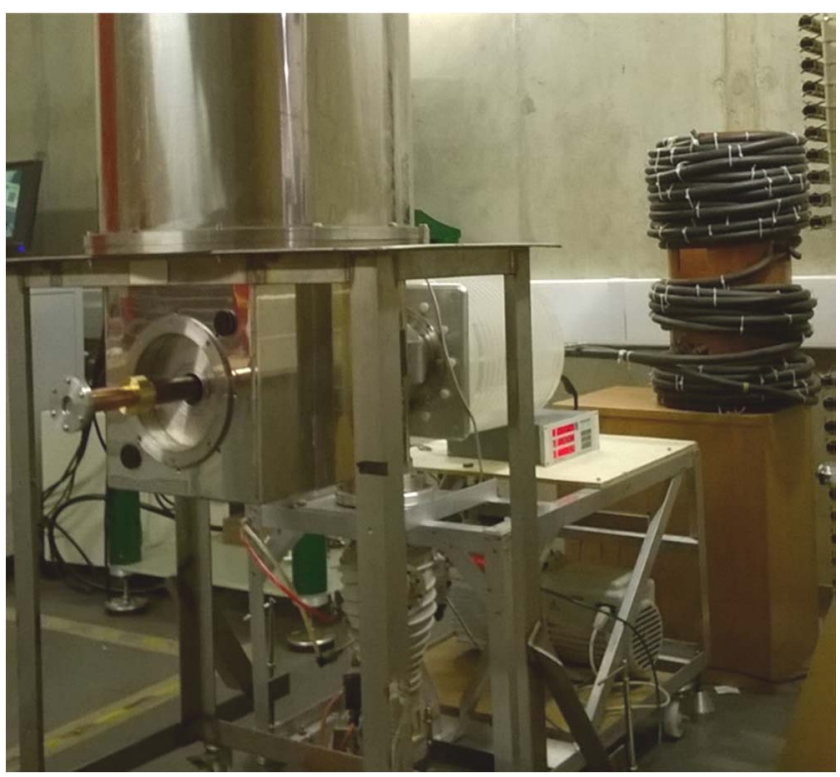

Fig. 3. Assembled oversized W-band 2D periodic lattice oscillator.

\section{ACKNOWLEDGMENT}

The authors thank David Barclay for his technical assistance.

\section{REFERENCES}

[1] R. Letizia, M. Mineo, and C. Paoloni, "Photonic Crystal-Structures for $\mathrm{THz}$ Vacuum Electron Devices", IEEE. Trans. Electron Devices, vol. 62, pp. 178-183, Jan. 2015.

[2] N. S. Ginzburg, N. Y. Peskov, A. S. Sergeev, et al. , "Theory of freeelectron maser with two-dimensional feedback driven by an annular electron beam", J. Appl. Phys., vol. 92, pp. 1619-1629, Aug. 2002.

[3] A. W. Cross, I. V. Konoplev, A. D. R. Phelps, and K. Ronald, "Studies of surface two-dimensional photonic band-gap structures", J. Appl. Phys., vol. 93, pp. 2208-2218, Feb. 2003.

[4] I. V. Konoplev, P. McGrane, A. D. R. Phelps, et al., "Observation of photonic band-gap control in one-dimensional Bragg structures," Appl. Phys. Lett., vol. 87, (12), art. no. 121104, Sept. 2005.

[5] I. V. Konoplev, L. Fisher, K. Ronald, et al., "Surface-field cavity based on a two-dimensional cylindrical lattice", Appl. Phys. Lett., vol. 96, (23), art. no. 231111, June 2010

[6] I. V. Konoplev, L. Fisher, A. W. Cross, et al., 'Surface wave Cherenkov maser based on a periodic lattice", Appl. Phys. Lett., vol. 96, (26), art. no. 261101, June 2010.

[7] I. V. Konoplev, L. Fisher, A. W. Cross, et al., "Excitation of surface field cavity and coherence of electromagnetic field scattering on twodimensional cylindrical lattice," Appl. Phys. Lett., vol. 97, (26), art. no. 261102, Dec. 2010.

[8] I. V. Konoplev, A. J. MacLachlan, C. W. Robertson, et al.,"Cylindrical periodic surface lattice as a metadielectric: concept of a surface-field Cherenkov source of coherent radiation", Phys. Rev. A, vol. 84, (01), art. no. 013826, July 2011.

[9] I. V. Konoplev, A. J. MacLachlan, C. W. Robertson, et al., "Cylindrical, periodic surface lattice - Theory, dispersion analysis and experiment", Appl. Phys. Lett., vol. 101, (12), art. no. 121111, Sept. 2012.

[10] I. V. Konoplev, A. R. Phipps, A. D. R. Phelps, et al., "Surface field excitation by an obliquely incident wave", Appl. Phys. Lett., vol. 102, (14), art. no. 141106, Apr. 2013.

[11] N. S. Ginzburg, A. M. Malkin, A. S. Sergeev and V. Zaslavsky, "Oversized co-axial and cylindrical surface-wave oscillators with twodimensional periodical grating (quasi-optical model)", J. Appl. Phys., vol. 113, (10), art. no. 104504, Mar. 2013.

[12] A. R. Phipps, A. J. MacLachlan, C. W. Robertson, et al., "Electron beam excitation of coherent sub-terahertz radiation in periodic structures manufactured by 3D printing," Nucl. Instrum. Methods Phys. Res. B, vol. 402, pp. 202-205, Apr. 2017.

[13] N. S. Ginzburg, E. V. Ilyakov, I. S. Kulagin, et al., "Theoretical and experimental studies of relativistic oversized Ka-band surface-wave oscillator based on 2D periodical corrugated structure", Phys. Rev. Accel. Beams, vol. 21, (8), art. no. 080701, Aug. 2018.

[14] A. J. MacLachlan, C. W. Robertson, A. W. Cross, et al., "Volume and surface mode coupling experiments in periodic surface structures for use in mm-THz high power radiation sources," AIP Advances, vol. 8, (10), art. no. 105115, Oct. 2018. 